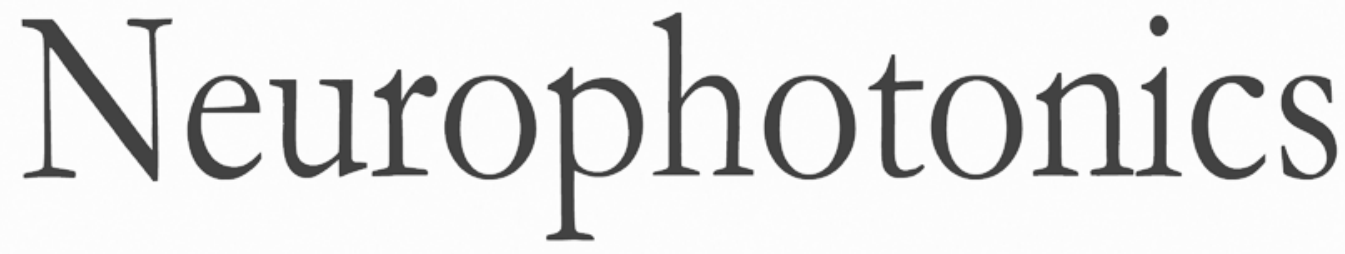

\title{
Spatial variation in the hemoglobin phase of oxygenation and deoxygenation in the developing cortex of infants
}

Gentaro Taga

Hama Watanabe

Fumitaka Homae 


\title{
Spatial variation in the hemoglobin phase of oxygenation and deoxygenation in the developing cortex of infants
}

\author{
Gentaro Taga, ${ }^{a, *}$ Hama Watanabe, ${ }^{a}$ and Fumitaka Homae ${ }^{b, c}$ \\ ${ }^{a}$ The University of Tokyo, Graduate School of Education, Tokyo, Japan \\ ${ }^{\mathrm{b} T}$ Tokyo Metropolitan University, Department of Language Sciences, Hachioji-shi, Tokyo, Japan \\ ${ }^{\mathrm{c}}$ Tokyo Metropolitan University, Research Center for Language, Brain and Genetics, Hachioji-shi, Tokyo, Japan
}

\begin{abstract}
Spontaneous low-frequency oscillatory changes in oxygenated hemoglobin (oxy- $\mathrm{Hb}$ ) and deoxygenated hemoglobin (deoxy- $\mathrm{Hb}$ ) are observed using functional near-infrared spectroscopy (fNIRS). A previous study showed that the time-averaged phase difference between oxy-Hb and deoxy-Hb changes, referred to as hemoglobin phase of oxygenation and deoxygenation (hPod), is sensitive to the development of the cortex. We examined phase-locking index of $\mathrm{hPod}$, referred to as $\mathrm{hPod}$, in addition to $\mathrm{hPod}$, in neonates and 3- and 6-month-old infants using the 94-channel fNIRS data, which covered large lateral regions of the cortex. The results showed that (1) developmental changes in hPod exhibited spatial dependency; (2) $\mathrm{hPod} \mathrm{L}_{\mathrm{L}}$ increased between the neonate group and 3-month-old infant group over the posterior, but not anterior, regions of the cortex; and (3) the cortical regions of each age group were clustered in several domains with specific characteristics of $\mathrm{hPod}$ and $\mathrm{hPod}$. This study indicates that the neonatal cortex is composed of regions with specific characteristics of $\mathrm{hPod}$ and $\mathrm{hPod}$, and drastic changes occur between the neonatal period and 3 months of age. This study suggests that $\mathrm{hPod}$ and $\mathrm{hPod}$ are sensitive to the cortical region-specific development of the circulatory, blood flow, metabolic, and neurovascular functions in young infants. ๑ 2017 Society of Photo-Optical Instrumentation Engineers (SPIE) [DOI: 10.1117/1.NPh.5.1.011017]
\end{abstract}

Keywords: infants; functional near-infrared spectroscopy; hemoglobin phase of oxygenation and deoxygenation; developing cortex; resting state.

Paper 17092SSR received Apr. 15, 2017; accepted for publication Sep. 18, 2017; published online Oct. 10, 2017.

\section{Introduction}

Spatiotemporal changes in spontaneous low-frequency oscillations of the cortical hemodynamics are observed in infants through multichannel functional near-infrared spectroscopy (fNIRS). ${ }^{1}$ The signals of relative changes in oxygenated hemoglobin (oxy-Hb) and deoxygenated hemoglobin (deoxy-Hb) concentrations in the cerebral tissue at multiple locations measured with fNIRS provide rich information to reveal the development of functional connectivity. ${ }^{2-4}$ The fNIRS has also been measured in neonates as a clinical tool for quantitatively assessing the brain development. ${ }^{5-10}$

A recent study with fNIRS showed that time-averaged phase differences between spontaneous low-frequency oscillatory changes in oxy- $\mathrm{Hb}$ and deoxy- $\mathrm{Hb}$ in the cerebral tissue showed dramatic changes from an in-phase to an antiphase pattern in the first six months of life. ${ }^{11}$ This phase difference is referred to as hemoglobin phase of oxygenation and deoxygenation (hPod). The study reported that hPod showed subtle differences in development between term- and preterm-born infants. Thus, hPod can be a good marker for determining the developmental stage of the integration of circulatory, blood flow, metabolic, and neurovascular functions in the brain, with high sensitivity.

While the hPod value in the previous study was obtained by averaging the signals of 10 channels covering specific regions of the cortex, ${ }^{11}$ the spatial differences in hPod were not well-studied. The spatial patterns of functional activity in the cortex in early infancy have been extensively reported. ${ }^{12-15}$ The development of functional connectivity between the specific regions of the cortex has been observed in the first few months of life. ${ }^{2}$ Thus, hPod may exhibit region-dependent differences that reflect functional development. Another issue that was not examined in the previous study ${ }^{11}$ is whether the phase differences between oxy- and deoxy-Hb changes are stable or fluctuating. To quantify this property, we assessed the degree of phase locking of hPod, referred to as $\mathrm{hPod}_{\mathrm{L}}$. In this study, we focused on the spatial properties of $h$ Pod and $h \mathrm{hod}_{\mathrm{L}}$ over large cortical regions using the existing data measured with a 94-channel fNIRS for studying functional connectivity. ${ }^{2}$

\section{Methods}

We analyzed the existing data of Homae et al. ${ }^{2}$ in this study. Here, we summarize the participants, apparatus, and procedures, and then describe the data analysis.

\subsection{Participants}

We analyzed the data from 52 full-term born infants who were sleeping naturally. They were divided into three different age groups. Fifteen were neonates ( 6 girls and 9 boys; mean postnatal age: 4.3 days, range: 2 to 11 days), 21 were 3 -month-old infants (11 girls and 10 boys; mean postnatal age: 111.6 days, 
range: 102 to 123 days), and 16 were 6-month-old infants (6 girls and 10 boys; mean postnatal age: 197.0 days, range: 180 to 206 days). Ethical approval for this study was obtained from the ethical committee of Tokyo Women's Medical University for studying the neonates and Graduate School of Education, University of Tokyo, for studying the 3- and 6-month-old infants. Written informed consent was obtained from the parent(s) of all the participants before the experiment. ${ }^{2}$

\subsection{Apparatus and Procedures}

A near-infrared spectroscopy (NIRS) instrument (ETG-7000; Hitachi Medical Corporation) with 94 measurement channels (47 channels in each hemisphere) was used to detect the relative concentration changes in oxy-Hb and deoxy-Hb [millimolar-millimeter $(\mathrm{mM} \cdot \mathrm{mm})]$ with a $0.1-\mathrm{s}$ resolution. This instrument uses two wavelengths of NIR light (785 and $830 \mathrm{~nm}$ ). NIR light was emitted from laser diodes through incident optical fibers (maximum intensity: $0.4 \mathrm{~mW}$ for neonates, $0.6 \mathrm{~mW}$ for 3 month-old infants, and $1.2 \mathrm{~mW}$ for 6-month-old infants). Two sets of $3 \times 10$ array probes composed of 15 sources and 15 detectors of NIR light were mounted on a flexible cap over the frontal, temporal, parietal, and occipital regions of each hemisphere. The distance between the source and detector was set to $\sim 2.0 \mathrm{~cm}$, and each pair of adjacent incident and detection fibers defined a single measurement channel. ${ }^{2}$

The measurement channels were positioned by referring to the international 10/20 system of electrode placement and using the external auditory pores, vertex, and inion as landmarks in each participant. The locations of 94 channels can be overlaid onto the magnetic resonance imaging brain atlas using the virtual registration method. ${ }^{16}$ Figure 1 shows a schematic presentation of estimated channel locations over the cortex based on the virtual registration. The channels cover the frontal pole (FP), superior frontal gyrus (SFG), middle frontal gyrus (MFG),

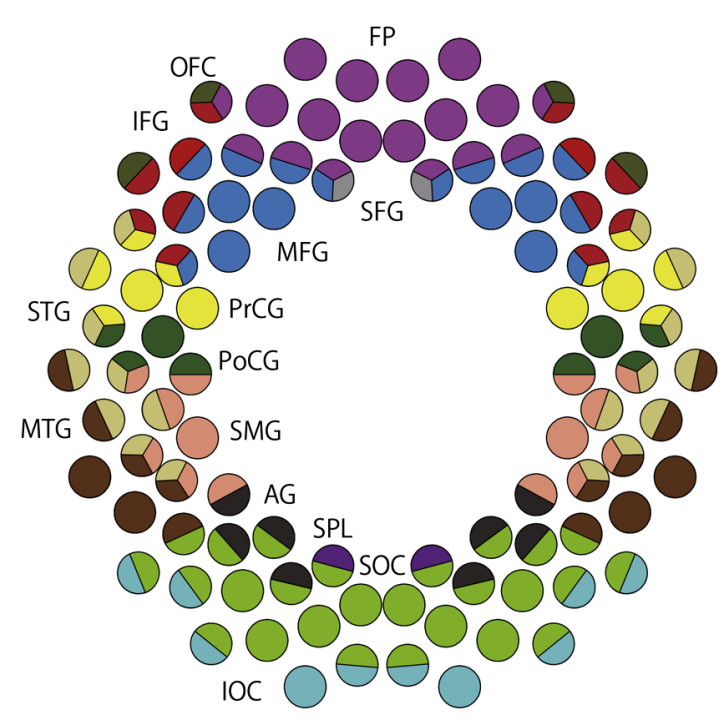

Fig. 1 Measurement channels over cortical regions. Circles indicate measurement channels, and colors denote the cortical regions as follows: FP, dark magenta; SFG, light gray; MFG, blue; IFG, red; OFC, olive; PrCG, yellow; PoCG, green; SMG, wheat; AG, dark gray; SPL, purple; STG, khaki; MTG, brown; SOC, light green; and IOC, light blue. Some channels, which were coded by two or three colors, detect signal changes from not a single cortical region. inferior frontal gyrus (IFG), orbitofrontal cortex (OFC), precentral gyrus (PrCG), postcentral gyrus (PoCG), supramarginal gyrus (SMG), angular gyrus (AG), superior parietal lobule (SPL), superior temporal gyrus (STG), middle temporal gyrus (MTG), and superior and inferior occipital cortices (SOC and IOC).

The measurements were obtained when the neonates or infants were in natural sleep in a dimly lit room.

\subsection{Data Analysis}

\subsubsection{Preprocessing}

We detected the periods containing motion artifacts in the oxy$\mathrm{Hb}$ and deoxy-Hb time series data from all 94 channels and excluded them from the analysis. When we detected changes in the sum of the oxy-Hb and deoxy-Hb signals of more than $0.15 \mathrm{mM} \cdot \mathrm{mm}$ in the mean of four successive samples (i.e., $400 \mathrm{~ms}$ ) and that of the next four successive samples, we regarded the last time point in the first four samples as the time at which a motion artifact occurred in the affected channel. We then labeled the data from the preceding 60 samples and those from the next 250 samples as a motion artifact period. If 47 or more channels (i.e., half of the channels) showed motion artifacts at the same time point, all the channels were assumed to be affected by motion artifacts at that time point. As a result of rejection of the motion artifacts, $97.6 \%$ of time point data on average among participants was used for analysis. Then, oxy$\mathrm{Hb}$ and deoxy-Hb time course signals were filtered through a frequency band of 0.05 to $0.1 \mathrm{~Hz}$ using a fast Fourier transform to remove physiological noise (e.g., heartbeats and respirations) and measurement noise (e.g., slow signal drifts and highfrequency noise). Note that the lower frequency $(0.05 \mathrm{~Hz})$ corresponds to the time scale of hemodynamic response to neural activity $(20 \mathrm{~s})$.

\subsubsection{Calculation of $h P o d$ and $h$ Pod $_{L}$}

The value of hPod was calculated according to the method by Watanabe et al. ${ }^{11}$ In short, a Hilbert transformation was applied to the preprocessed time series of oxy-Hb and deoxy-Hb for each channel to obtain the instantaneous phases of each signal. The phase difference between the instantaneous phases of oxy$\mathrm{Hb}$ and deoxy-Hb signals was calculated and represented by a vector with a phase value and length of 1 . The time series of vectors were averaged over the measurement time. We finally obtained the averaged values of phase and length for each of the 94 channels. The averaged value of phase was termed hPod. ${ }^{11}$ The average value of length represents the degree of phase locking between oxy-Hb and deoxy-Hb and was referred to as $\mathrm{hPod}_{\mathrm{L}}$ in this study, as shown in Fig. 2(a). An angular value of hPod larger than $1.5 \pi$ indicates a pattern close to an in-phase one, while a value smaller than $1.5 \pi$ indicates a pattern close to an antiphase one. A scalar value of $\mathrm{hPod}_{\mathrm{L}}$ close to 1 indicates high phase locking, while a value close to zero indicates little phase locking or large fluctuation of phase.

\subsubsection{Statistical analysis}

Figure 2(b) shows how group averaging was performed to be subjected to group analysis for each of the hPod and $h \operatorname{Pod}_{L}$. To investigate the developmental changes in the vector values of hPod in each location of the cortex, the Watson-Williams test was applied to the hPod values of the three age groups on a channel-by-channel basis. To compare the hPod values 
(a)

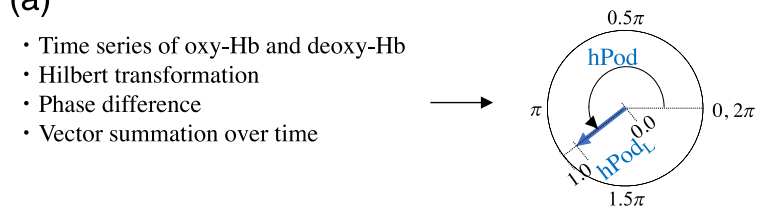

(b)
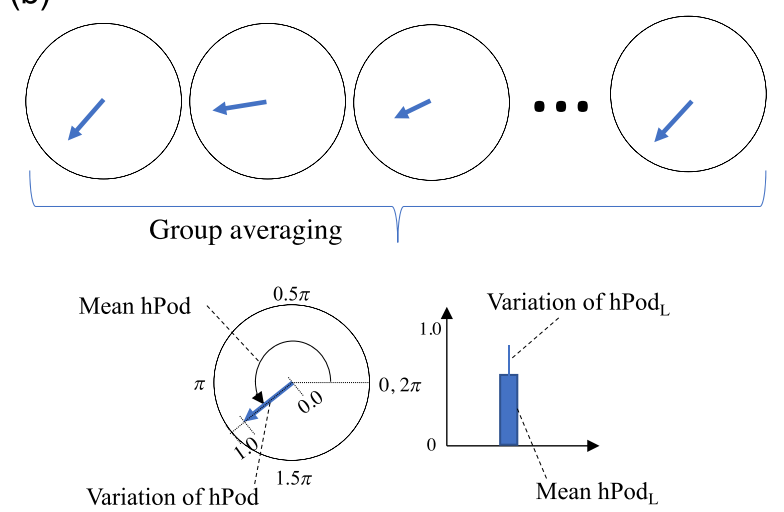

Fig. 2 Definition of $h P o d$ and $h$ Pod $_{L}$ : (a) hPod and hPod ${ }_{L}$ obtained from each channel of an individual and (b) group analysis of hPod and $\mathrm{hPod}$.

among the three age groups, the Tukey-Welsch procedure was used (Watanabe et al. ${ }^{11}$ for details of circular statistics). We used the Bonferroni correction for the number of channels to account for multiple testing and considered significance when $p<0.05 / 94=0.00053$.

To investigate the developmental changes in the scalar values of $\mathrm{hPod}_{\mathrm{L}}$ in each location of the cortex, the analysis of variance (ANOVA) of the $\mathrm{hPod}_{\mathrm{L}}$ values of the three age groups was performed on a channel-by-channel basis. To conduct the comparisons among the age groups, a $t$-test with Ryan's method was performed. Bonferroni correction for the number of channels was used for the repetition of testing on a channelby-channel basis. Thus, the cut-off level of significance was set at $p<0.05 / 94=0.00053$.

\subsubsection{Cluster analysis}

To reveal the spatial properties of $h P o d$ and $h \operatorname{Pod}_{L}$ in each age group, the group mean values of the two variables at all 94 channels were subjected to cluster analyses. The phase values of hPod were assumed to be scalar because the distribution of hPod values in each age group was thought to be concentrated within the range of $\pi$ and $2 \pi$. Prior to clustering the data, the two variables were normalized. A hierarchical clustering with the Ward method generated a dendrogram. The tree of the dendrogram was cut at a specific height of the distance between the clusters, and a specific number of clusters were obtained. The clustered channels were topographically mapped on the estimated standard cortical regions of the channels as shown in Fig. 1. To validate and interpret the clustering results, we examined whether the clusters were distinguishable by comparing the clustered means in $\mathrm{hPod}$ and $\mathrm{hPod}_{\mathrm{L}}$ using ANOVA. Note that phase variables in hPod were treated as scalar values, since the variables were considered to be narrowly distributed within each age group. To conduct the comparisons among the clusters, a $t$-test with Ryan's method was performed. The cut-off level of significance was set at $p<0.05$.
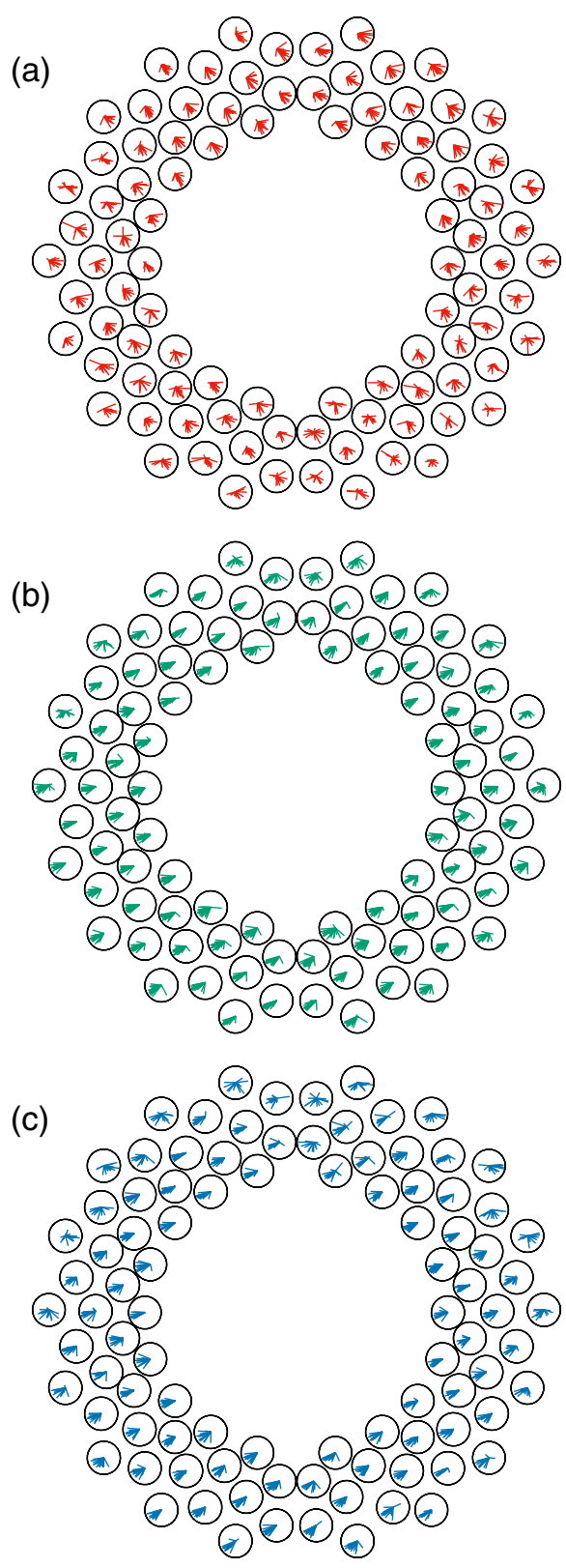

Fig. 3 Vector representation of $\mathrm{hPod}$ and $\mathrm{hPod}$ for each of the 94 channels from individual infants in different age groups: (a) neonates (red), (b) 3-month-olds (green), and (c) 6-month-olds (blue).

\section{Results}

Figure 3 shows a vector presentation of the individual values of hPod (direction) and $\mathrm{hPod}_{\mathrm{L}}$ (length) for each channel, which were superimposed in each age group. In the neonate group, the hPod values ranged between $1.5 \pi$ and $2 \pi$ at most of the channels, indicating that the hPod pattern was close to the in-phase one. In contrast, the hPod values ranged between $\pi$ and $1.5 \pi$ at most of the channels of the 3- and 6-month-old infant groups, respectively, indicating that the hPod pattern was close to antiphase. The $h \operatorname{Pod}_{\mathrm{L}}$ values in the neonate groups were small overall (short vectors), indicating low phase locking of hPod. In contrast, $\mathrm{hPod}_{\mathrm{L}}$ values in the 3- and 6-month-old infant groups overall showed high phase locking (long vectors).

To clarify the properties of $\mathrm{hPod}$ and $\mathrm{hPod}_{\mathrm{L}}$ in each age group and differences in these values among the age groups, 
(a)

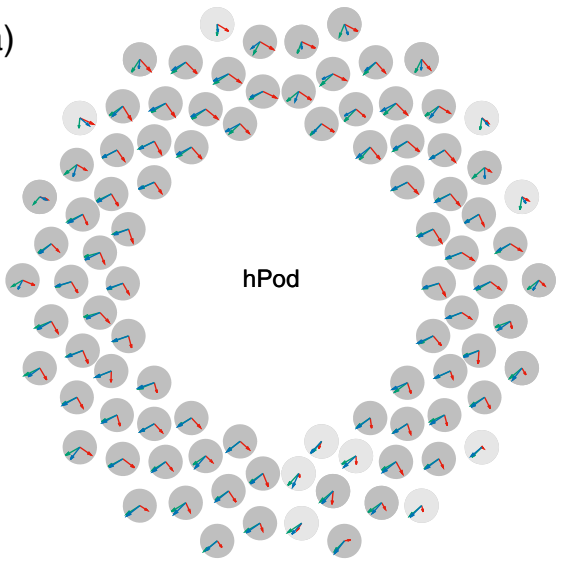

(b)

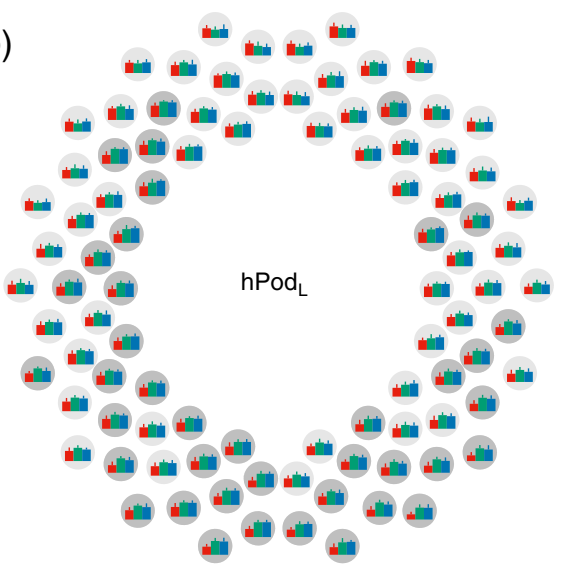

Fig. 4 Developmental changes in the group means and variations for values of $h P o d$ and $h P o d_{L}$ in each channel. (a) Group means and variations of $\mathrm{hPod}$. The direction of a vector represents $\mathrm{hPod}$, and the length represents an interinfant variation in hPod value. Colored arrows with red, green, and blue, show data from the neonate, 3-month-old, and 6-month-old infant groups, respectively. (b) Group means and variations of $h$ Pod $_{L}$. A bar represents a value of $h P o d_{L}$, and an error bar represents a value of interinfant variation. Colored bars with red, green, and blue show data from the neonate, 3-month-old, and 6-month-old infants, respectively.

the mean values and variations were calculated for each hPod and $\mathrm{hPod}_{\mathrm{L}}$ values on a channel-by-channel basis, as shown in Fig. 4. Note that the means of hPod values were obtained on the basis of vector calculation, and the length of the vectors represented the interinfant variation of the phase difference in each channel within the age group. The development of the mean hPod value from neonates to 3- to 6-month-old infants was observed in each location of the channel, as shown in Fig. 4(a). The Watson-Williams test to the hPod values revealed that the main effects of the age group were significant at 84 out of 94 channels, marked in dark gray in Fig. 4(a). Of the 84 channels, significant differences were observed between neonates and 3-month-old infants and between neonates and 6-month-old infants in 73 channels. At the remaining 11 channels, significant differences were observed only between neonates and 3-monthold infants. These findings indicate that the major changes in the hPod values occur between birth and 3 months of age over most of the cortical regions.

The development of the scalar values of $\mathrm{hPod}$ 和as assessed as shown in Fig. 4(b). The mean values of $\mathrm{hPod}_{\mathrm{L}}$ of the three groups were illustrated in three bars at each channel location, and the interinfant variations of $\mathrm{hPod}_{\mathrm{L}}$ values were also shown as error bars on the bars [Fig. 4(b)]. The ANOVA using the $\mathrm{hPod}_{\mathrm{L}}$ values revealed that the main effects of the age group were significant at 40 out of 94 channels, marked in dark gray. Of the 40 channels, 25 showed significant differences between neonates and 3-month-old infants and between neonates and 6-month-old infants, 13 showed significant differences only between neonates and 3-month-old infants, and 2 showed significant differences only between neonates and 6-month-old infants. Interestingly, the channels on the posterior regions of the cortex showed significant agerelated changes as compared with the channels on the anterior regions of the cortex. The channels at the FP showed relatively high $\mathrm{hPod}_{\mathrm{L}}$ values in the neonate group and no significant developmental changes. These findings indicate that the major changes in $\mathrm{hPod}_{\mathrm{L}}$ values occurred between birth and 3 months and that a part of the anterior region of the cortex showed different development from the posterior region of the cortex.

To reveal the spatial differences in $\mathrm{hPod}$ and $\mathrm{hPod}$ of each age group, the mean values of $\mathrm{hPod}$ and $\mathrm{hPod}_{L}$ of each channel are illustrated by colored circles, with the color representing a phase value of hPod and the size representing a value of $h \operatorname{Pod}_{\mathrm{L}}$, as shown in Fig. 5. Overall, the change in color from red to blue occurred between the neonates and the 3-month-old infants at most of the channels, indicating that the major changes in hPod occurred during this period regardless of the cortical regions. Careful assessment of the spatial pattern of colored circles at each age revealed cortical region-dependent differences. We examined whether the channels could be spatially clustered based on hPod and $\mathrm{hPod}$. The hierarchical clustering with the Ward method generated a dendrogram for each age group. We cut the tree of the dendrograms at the same height of 10.0 in the three groups. The channels were then clustered into 5, 4, and 3 for the neonates, 3-, and 6-month-old infant groups, respectively.

In the neonates, the channels were clustered into 1a $\left(\mathrm{hPod}=1.78 \pi, \mathrm{hPod}_{\mathrm{L}}=0.63\right.$, number of channel $\left.n=27\right)$, $1 \mathrm{~b}\left(\mathrm{hPod}=1.86 \pi, \mathrm{hPod}_{\mathrm{L}}=0.53, n=13\right), 2 \mathrm{a}(\mathrm{hPod}=1.68 \pi$, $\left.\mathrm{hPod}_{\mathrm{L}}=0.53, n=29\right), 2 \mathrm{~b} 1\left(\mathrm{hPod}=1.69 \pi, \mathrm{hPod}_{\mathrm{L}}=0.38\right.$, $n=8)$, and $2 \mathrm{~b} 2\left(\mathrm{hPod}=1.54 \pi, \mathrm{hPod}_{\mathrm{L}}=0.48, n=17\right)$. The ANOVA using the hPod values revealed that the main effect of the cluster was significant $(p<0.001)$. The post-hoc comparisons among the clusters revealed that all the combinations except for the one between $2 \mathrm{a}$ and $2 \mathrm{~b} 1$ were significantly different $(p<0.05)$. The ANOVA using the $\mathrm{hPod}_{\mathrm{L}}$ values revealed that the main effect of the cluster was significant $(p<0.001)$. The post-hoc comparisons among the clusters revealed that all the combinations except for the one between $2 \mathrm{a}$ and $1 \mathrm{~b}$ were significantly different $(p<0.05)$.

In 3-month-old infants, the channels were clustered into 1a $\left(\mathrm{hPod}=1.39 \pi, \mathrm{hPod}_{\mathrm{L}}=0.51, n=10\right), 1 \mathrm{~b}(\mathrm{hPod}=1.22 \pi$, $\left.\mathrm{hPod}_{\mathrm{L}}=0.68, n=20\right), 2 \mathrm{a}\left(\mathrm{hPod}=1.18 \pi, \mathrm{hPod}_{\mathrm{L}}=0.80\right.$, $n=30)$, and $2 \mathrm{~b}\left(\mathrm{hPod}=1.13 \pi, \mathrm{hPod}_{\mathrm{L}}=0.76, n=34\right)$. The ANOVA using the hPod values revealed that the main effect of the cluster was significant $(p<0.001)$. The post-hoc comparisons among the clusters revealed that all the combinations were significantly different $(p<0.05)$. The ANOVA using the $\mathrm{hPod}_{\mathrm{L}}$ values revealed that the main effect of the cluster was significant $(p<0.001)$. The post-hoc comparisons among the clusters revealed that all the combinations were significantly different $(p<0.05)$. 

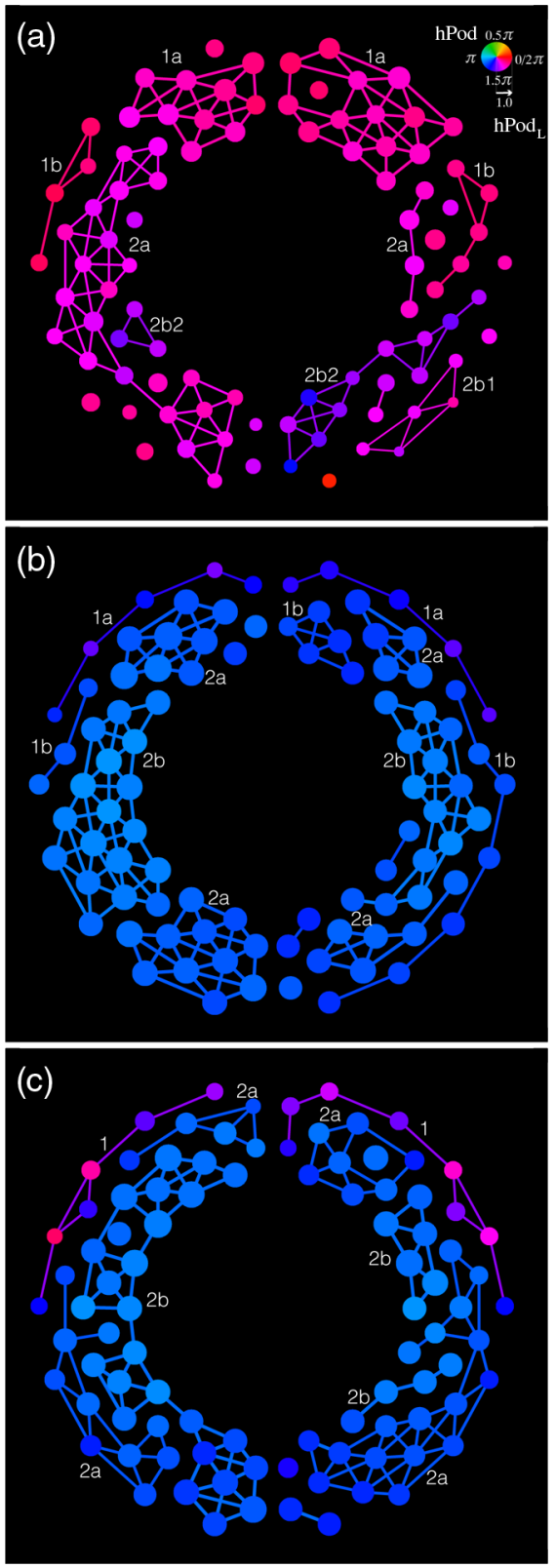

Fig. 5 Spatial clustering of $\mathrm{hPod}$ and $\mathrm{hPod}$. Color of a circle represents the mean value of $\mathrm{hPod}$, and the size represents the mean value of $\mathrm{hPod}_{\mathrm{L}}$ for each channel. (a) Neonates: the channels were clustered into five (1a, 1b, 2a, 2b1, and 2b2). (b) 3-month-olds: the channels were clustered into four (1a, 1b, 2a, and 2b). (c) 6month-olds: the channels were clustered into three $(1,2 a$, and $2 b)$. The lines connecting the channels were drawn when the spatially adjacent channels belonged to the same cluster. The color and width of the lines connecting channels were determined according to the mean value of $\mathrm{hPod}$ and $\mathrm{hPod}$, respectively, within the same cluster.

In 6-month-old infants, the channels were clustered into 1 $\left(\mathrm{hPod}=1.53 \pi, \operatorname{hPod}_{\mathrm{L}}=0.55, n=15\right), 2 \mathrm{a}(\mathrm{hPod}=1.20 \pi$, $\left.\mathrm{hPod}_{\mathrm{L}}=0.67, n=41\right)$, and $2 \mathrm{~b}\left(\mathrm{hPod}=1.16 \pi, \mathrm{hPod}_{\mathrm{L}}=0.78\right.$, $n=38$ ). The ANOVA using the hPod values revealed that the main effect of the cluster was significant $(p<0.001)$. The post-hoc comparisons among the clusters revealed that all the combinations except for the one between $2 a$ and $2 b$ were significantly different $(p<0.05)$. The ANOVA using the $\mathrm{hPod}_{\mathrm{L}}$ values revealed that the main effect of the cluster

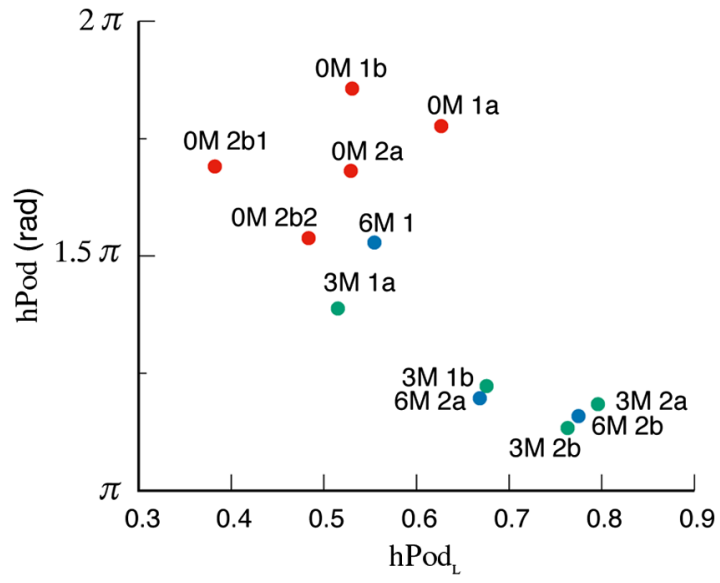

Fig. 6 Mean hPod versus $\mathrm{hPod}_{\mathrm{L}}$ for each cluster. Data of clustered channels of neonates (1a, 1b, 2a, 2b1, and 2b2), 3-month-old infants (1a, 1b, 2a, and 2b), and 6-month-old infants (1, 2a, and 2b) were shown in red, green, and blue, respectively.

was significant $(p<0.001)$. The post-hoc comparisons among the clusters revealed that all the combinations were significantly different $(p<0.05)$.

As shown in Fig. 5, the lines connecting channels were drawn when the spatially adjacent channels belonged to the same cluster; thereby, spatial continuity of clusters was observable. Figure 6 shows plotting of the mean values of hPod versus $\mathrm{hPod}_{\mathrm{L}}$ for each cluster for each age group.

\section{Discussion}

This study found that the hPod values decreased from an in-phase to an antiphase pattern between the newborn period and 3 months of age at most of the lateral cortical regions that the 94-channel fNIRS probe could cover [Fig. 4(a)]. This result is consistent with the previous report in which the termborn infants showed a rapid change in hPod occurring at birth and a gradual change in hPod lasting during the first 6 months of age. ${ }^{11}$ However, the typical developmental pattern was not observed at some of the channels on the ventral side of the frontal lobe and right posterior regions. Thus, this study revealed that the development of hPod has a cortical region specificity.

This study focused not only on the hPod values but also on the $h \operatorname{Pod}_{\mathrm{L}}$ values, which represent the degrees of phase locking of hPod. While the value of hPod has been suggested to be a good indicator of the developmental changes in dominant physiological mechanisms underlying the circulatory, blood flow, metabolic, and neurovascular functions in the previous study, ${ }^{11}$ the value of $\mathrm{hPod}_{\mathrm{L}}$ provides additional and important information on the strength of the coupling among the related mechanisms, such as the blood flow and metabolism coupling and the neurovascular coupling. Even when the hPod values are similar, $\mathrm{hPod}_{\mathrm{L}}$ values could represent differences in stability of interaction among the physiological processes. We found that the development of $\mathrm{hPod}_{\mathrm{L}}$ significantly differed on the basis of the cortical regions [Fig. 4(b)]. In the posterior cortical regions, several channels showed a significant increase in $\mathrm{hPod}_{\mathrm{L}}$ between the newborn period and 3 months of age. In the anterior cortical regions, several channels tended to show large $\mathrm{hPod}_{\mathrm{L}}$ values in the newborn period, and no significant increase in $\mathrm{hPod}_{\mathrm{L}}$ values was observed. These data indicate that the development of the phase-locking property of hPod differed on the 
basis of the cortical regions. The cortical region-dependent development was more prominent in the $\mathrm{hPod}_{\mathrm{L}}$ than in the $\mathrm{hPod}$.

The cluster analysis of $\mathrm{hPod}$ and $\mathrm{hPod}_{\mathrm{L}}$ (Fig. 5) with the estimated cortical regions (Fig. 1) revealed precise spatial differences. The statistical analysis showed that clusters based on the combination of $\mathrm{hPod}$ and $\mathrm{hPod}_{\mathrm{L}}$ in each age group are distinguishable. Neonates already showed cortical regionspecific characteristics. The FP and MFG of the neonates showed an in-phase pattern with high phase locking (cluster 1a), which were distinguished from the characteristics of other regions. Cluster 2a was distinguishable from cluster $1 \mathrm{~b}$ based on hPod and from cluster $2 \mathrm{~b} 1$ based on $\mathrm{hPod}_{\mathrm{L}}$. In 3-month-old infants, the temporal regions, including the STG and MTG, a part of the frontal region, including the PrCG, and the parietal region, including the PoCG and SMG, bilaterally constituted a distinct cluster with antiphase pattern and high phase locking (cluster 2b). Bilateral frontal regions, including the FP and MFG, and bilateral occipital regions belonged to the same cluster with antiphase pattern and high phase locking (cluster 2a). The ventral parts of the frontal, temporal, and occipital regions showed an hPod value close to $1.5 \pi$ and low phase locking (cluster 1a and 1b). The 6-month-old infants showed a spatial pattern with a similar topology of clusters as those of the 3-month-old infants, although the form and size of each cluster in the 6-month-old group were different from those of the 3-month-old group. The ventral portions of the frontal and temporal regions showed an hPod value more than $1.5 \pi$ (cluster 1), which was very different from the characteristics of the other two clusters. Overall, each cortical region exhibited a specific pattern of development of hPod and $\mathrm{hPod}_{\mathrm{L}}$. For instance, the cortical region in the FP and MFG of the left hemisphere in the neonate group was classified to a single cluster with an in-phase pattern with high phase locking. However, the same region was differentiated into two clusters, an antiphase pattern with high phase locking and a $1.5 \pi$-phase pattern with low phase locking, in the 3-month-old infant group. This region was further differentiated into three clusters, an antiphase pattern with high phase locking, an antiphase pattern with low phase locking, and $1.5 \pi$-phase pattern with low phase locking, in the 6-month-old infant group. The bilateral temporal regions around the STG showed an in-phase pattern with low phase locking in the neonates group, and this changed to an antiphase pattern with high phase locking in the 3-month-old infants.

Watanabe et al. ${ }^{11}$ used a model by Fantini ${ }^{17}$ with Hill's equation for hemoglobin oxygen saturation to estimate the changes in concentration of oxy- $\mathrm{Hb}$ and deoxy- $\mathrm{Hb}$ in the artery, capillary, and vein. The analysis of the model showed how physiological parameters, such as partial pressure of oxygen, partial blood volume, oxygen utilization rate, and speed of blood flow, affect the in-phase or antiphase pattern of hPod. The following scenario for the development of hPod was suggested. ${ }^{11}$ The high values of hPod in the newborn period may be accounted for by a dominance of the in-phase contributions of the partial blood volume. The rapid initial decrease in the hPod value may be induced by a rapid increase in the antiphase contribution of the partial pressure of oxygen, which is affected by the transition from fetal to adult $\mathrm{Hb}$. The gradual decrease in the hPod value toward an antiphase pattern after 3 months of age may reflect the increasing antiphase contribution of the speed of blood flow in the capillaries. The overall development of the complete lateral regions of the cortex can be accounted for by the aforementioned scenario of hPod development. However, the differences in $\mathrm{hPod}_{\mathrm{L}}$ in addition to hPod among the cortical regions that were found at each age in this study revealed the evidence to suggest spatially differential development of the circulatory, blood flow, metabolic, and neurovascular functions.

Regarding the development of metabolism, the method of magnetic resonance spectroscopy has been used to reveal that the cortical regions show drastic changes in metabolism between birth and 3 months of age. ${ }^{18}$ The cerebral metabolic rate of oxygen $\left(\mathrm{CMRO}_{2}\right)$ also may undergo drastic increases as suggested by a positron emission tomography (PET) study with a limited number of participants. ${ }^{19}$ Although $\mathrm{hPod}$ and $\mathrm{hPod}_{\mathrm{L}}$ may reflect changes in metabolism, hPod and $\mathrm{hPod}_{\mathrm{L}}$ are based not on the absolute values of the baseline of $\mathrm{CMRO}_{2}$ but on the timing of the hemodynamic changes. While both the changes in blood flow and $\mathrm{CMRO}_{2}$ contribute to an antiphase pattern of $\mathrm{hPod}$, their effects may be cancelled due to their opposing contribution to oxygen concentration. Thus, if the increase in blood flow was regulated to outpace the increase in $\mathrm{CMRO}_{2}$, the effect of the blood flow on hPod would be dominant. However, if the changes in blood flow and $\mathrm{CMRO}_{2}$ were decoupled, $\mathrm{hPod}_{\mathrm{L}}$ would show a low value, indicating unlocking of the phase. The anterior regions of neonates showed higher phase locking (cluster 1a), but other regions showed lower phase locking. In contrast, the anterior regions of the older groups showed lower phase locking (cluster 1a for 3 month and cluster 1 for 6 month), but the temporal and posterior regions showed higher phase locking. A possible physiological interpretation for the developmental inversion is as follows. In neonates, the anterior regions are dominated by changes in blood flow with low oxygen consumption, leading to higher phase locking. The temporal and posterior regions may undergo functional development requiring more oxygen consumption, but the coupling between the blood flow and oxygen metabolism is not established, leading to lower phase locking. In older ages, the blood flow and metabolism coupling and the neurovascular coupling are established in the temporal and posterior regions, leading to higher phase locking. However, the anterior regions may undergo prolonged development in comparison with other regions, leading to lower phase locking. Thus, $\mathrm{hPod}_{\mathrm{L}}$ should provide information on the balance between the blood flow and oxygen metabolism and the neurovascular coupling in each region of the cortex during development.

While many studies on brain glucose metabolism focused on oxidative phosphorylation, aerobic glycolysis occurs in the adult brain. ${ }^{20}$ In particular, aerobic glycolysis is significantly elevated in the medial and lateral parietal and prefrontal cortices and may play important roles in the cortical region-specific demands for energy consumption and biosynthesis-related synapse formation and growth. ${ }^{21}$ A study using PET showed that energy requirements of the fetal and newborn brain were fulfilled through a nonoxidative mechanism. ${ }^{22}$ Although data on the distribution of aerobic glycolysis in the brain are not available in young infants, it is important to know whether the spatial differences in $\mathrm{hPod}$ and $\mathrm{hPod}_{\mathrm{L}}$ are related to the form of metabolism that is vastly different from that in adults. If aerobic glycolysis induces the blood flow, the in-phase pattern of hPod, as was observed in the newborn group, may be obtained, which is an issue to be clarified in a future study.

Numerous issues are yet to be solved. First, infants in this study were in the state of quiet sleep, which was determined on the basis of the absence of body movements, and it is unknown whether hPod and $\mathrm{hPod}_{\mathrm{L}}$ changes in accordance 
with the sleep states. An NIRS study with adults suggested that sleep states affect hPod. ${ }^{23}$ Since sleep is related to metabolic changes, hPod and $h \operatorname{Pod}_{\mathrm{L}}$ may be affected by circadian rhythms. Second, it is unknown whether $\mathrm{hPod}$ and $\mathrm{hPod}_{\mathrm{L}}$ differ depending on sensory stimulus conditions. It was shown that a taskinduced activity of the brain can be better detected by the phase information between oxy-Hb and deoxy-Hb changes. ${ }^{24,25}$ It would be important to clarify how the stimulus-induced activity and spontaneous activity produce differentiated values of $\mathrm{hPod}$ and $\mathrm{hPod}_{\mathrm{L}}$ on functionally different regions of the cortex. Third, it is not clear whether hPod and $\mathrm{hPod}_{\mathrm{L}}$ may depend on other parameters for fNIRS measurement, such as the depth of the measured volume from the head ${ }^{26}$ and the frequency of signals. $^{11,27}$

\section{Disclosures}

The authors have no relevant financial interests in the paper and no other potential conflicts of interest to disclose.

\section{Acknowledgments}

This work was partly supported by the Japan Society for Promotion of Science Grants-in-Aid for Scientific Research 26220004 (to G.T.), 26350924 (to H.W.), and 21613006 (to F.H.).

\section{References}

1. G. Taga et al., "Spontaneous oscillation of oxy-and deoxy-hemoglobin changes with a phase difference throughout the occipital cortex of newborn infants observed using non-invasive optical topography," Neurosci. Lett. 282(1), 101-104 (2000).

2. F. Homae et al., "Development of global cortical networks in early infancy," J. Neurosci. 30(14), 4877-4882 (2010).

3. B. R. White et al., "Bedside optical imaging of occipital resting-state functional connectivity in neonates," NeuroImage 59(3), 2529-2538 (2012).

4. M. Imai et al., "Functional connectivity of the cortex of term and preterm infants and infants with Down's syndrome," NeuroImage 85, 272-278 (2014).

5. B. Molavi et al., "Analyzing the resting state functional connectivity in the human language system using near infrared spectroscopy," Front. Hum. Neurosci. 7, 921 (2014).

6. M. A. Franceschini et al., "Assessment of infant brain development with frequency-domain near-infrared spectroscopy," Pediatr. Res. 61, 546-551 (2007).

7. I. Kato et al., "Extrauterine environment influences spontaneous low-frequency oscillations in the preterm brain," Brain Dev. 35(1), 17-25 (2013).

8. S. L. Ferradal et al., "Functional imaging of the developing brain at the bedside using diffuse optical tomography," Cereb. Cortex 26(4), 1558-1568 (2015).

9. G. Bale et al., "Relationship between cerebral cytochrome-c-oxidase and oxygenation is associated with brain injury severity in birth asphyxiated infants," in Clinical and Translational Biophotonics, Optical Society of America (2016).

10. A. Stammwitz et al., "Can the assessment of spontaneous oscillations by near infrared spectrophotometry predict neurological outcome of preterm infants?" in Oxygen Transport to Tissue XXXVII, pp. 521-531, Springer, New York (2016).

11. H. Watanabe et al., "Hemoglobin phase of oxygenation and deoxygenation in early brain development measured using fNIRS," Proc. Natl. Acad. Sci. U. S. A. 114(9), E1737-E1744 (2017).
12. G. Taga et al., "Brain imaging in awake infants by near-infrared optical topography," Proc. Natl. Acad. Sci. U. S. A. 100, 10722-10727 (2003).

13. F. Homae et al., "The right hemisphere of sleeping infant perceives sentential prosody," Neurosci. Res. 54, 276-280 (2006).

14. H. Watanabe et al., "Functional activation in diverse regions of the developing brain of the human infant," NeuroImage 43, 346-357 (2008).

15. H. Sato et al., "Cerebral hemodynamics in newborn infants exposed to speech sounds: a whole-head optical topography study," Hum. Brain Mapp. 33(9), 2092-2103 (2012).

16. M. Matsui et al., "Referential framework for transcranial anatomical correspondence for fNIRS based on manually traced sulci and gyri of an infant brain," Neurosci. Res. 80, 55-68 (2014).

17. S. Fantini, "A haemodynamic model for the physiological interpretation of in vivo measurements of the concentration and oxygen saturation of haemoglobin," Phys. Med. Biol. 47(18), N249-N257 (2002).

18. S. Blüml et al., "Metabolic maturation of the human brain from birth through adolescence: insights from in vivo magnetic resonance spectroscopy," Cereb. Cortex 23(12), 2944-2955 (2013).

19. H. T. Chugani, M. E. Phelps, and J. C. Mazziotta, "Positron emission tomography study of human brain functional development," Ann. Neurol. 22(4), 487-497 (1987).

20. S. N. Vaishnavi et al., "Regional aerobic glycolysis in the human brain," Proc. Natl. Acad. Sci. U. S. A. 107(41), 17757-17762 (2010).

21. M. S. Goyal et al., "Aerobic glycolysis in the human brain is associated with development and neotenous gene expression," Cell Metab. 19(1), 49-57 (2014).

22. D. I. Altman et al., "Cerebral oxygen metabolism in newborns," Pediatrics 92(1), 99-104 (1993).

23. M. L. Pierro et al., "Phase-amplitude investigation of spontaneous lowfrequency oscillations of cerebral hemodynamics with near-infrared spectroscopy: a sleep study in human subjects," NeuroImage 63(3), 1571-1584 (2012).

24. K. Yoshino and T. Kato, "Vector-based phase classification of initial dips during word listening using near-infrared spectroscopy," NeuroReport 23(16), 947-951 (2012).

25. G. Taga et al., "Spatiotemporal properties of cortical haemodynamic response to auditory stimuli in sleeping infants revealed by multi-channel near-infrared spectroscopy," Philos. Trans. R. Soc. A 369(1955), 4495-4511 (2011).

26. T. Funane et al., "Greater contribution of cerebral than extracerebral hemodynamics to near-infrared spectroscopy signals for functional activation and resting-state connectivity in infants," Neurophotonics 1(2), 025003 (2014).

27. S. Sasai et al., "Frequency-specific functional connectivity in the brain during resting state revealed by NIRS," NeuroImage 56, 252-257 (2011).

Gentaro Taga is a professor at the Graduate School of Education, the University of Tokyo, Japan. His research broadly focuses on the early development of the brain and behavior in humans.

Hama Watanabe is a project associate professor at the Graduate School of Education, the University of Tokyo, Japan. She is currently involved in studies for infant behavioral and neurological developments. She contributes over broad topics ranging behavioral science, psychological science, cognitive science, developmental science, and neuroscience.

Fumitaka Homae is an associate professor in the Department of Language Sciences, Tokyo Metropolitan University, Japan. His research focuses on developmental brain science of language. His research interests also include structural and functional development of cortical networks in the infant and toddler brain. 